\title{
The Effects of Selective Depletion of Hydrochloric Acid on Acid-Base and Electrolyte Equilibrium *
}

\author{
Mark A. Needle, George J. Kaloyanides, and William B. Schwartz \\ (From the Department of Medicine, Tufts University School of Medicine, and the Renal \\ Laboratory, Pratt Clinic-New England Center Hospital, Boston, Mass.)
}

Previous studies have demonstrated that hypochloremic alkalosis in the dog cannot be repaired unless the diet contains significant quantities of chloride $(1,2)$. It has also recently been shown that chloride depletion induced by infusion of sodium nitrate will lead to the development of a metabolic alkalosis that depends on chloride availability for its correction (3). In this latter study the possible specific effects of nitrate on potassium balance and on renal tubular function have complicated the interpretation of the results.

The present study was undertaken to evaluate the effects of extrarenal depletion of chloride on acid-base and electrolyte equilibrium. Selective removal of hydrochloric acid by gastric drainage has been chosen as the most physiologic means of achieving this goal. In contrast to previous studies (4-6), the possible effects of other extrarenal deficits have been avoided by replacement of the sodium chloride, potassium chloride, and fluid withdrawn from the stomach each day. The data demonstrate that removal of hydrochloric acid elevates the renal threshold for bicarbonate, accelerates sodium-hydrogen and sodium-potassium exchange, and induces a metabolic alkalosis that can be repaired only when the diet is supplemented by chloride.

\section{Materials and Methods}

Seven balance studies were carried out on six healthy female mongrel dogs weighing from 12 to $14 \mathrm{~kg}$. At least 10 days before the beginning of the metabolic study, each animal was subjected to a gastrostomy, and a nylon prosthesis was placed in the stomach at a point approximately $5 \mathrm{~cm}$ from the pylorus and $5 \mathrm{~cm}$ from the

* Submitted for publication December 20, 1963; accepted June 2, 1964.

Supported in part by grants HE-00759 and HTS 5309 from the National Heart Institute, U. S. Public Health Service, and by the Samuel Bass Fund for Kidney Research. greater curvature. ${ }^{1}$ A nylon plug was used to prevent leakage of gastric contents. Selective depletion of hydrochloric acid was accomplished in the following manner: In the early morning the fasting animal was given a subcutaneous injection of $25 \mathrm{mg}$ of Histalog ${ }^{\text {T.M. }}$ (betazole hydrochloride). ${ }^{2}$ Immediately thereafter the plug in the prosthesis was replaced by a plastic bag and the fluid collected for approximately 2 hours. The animal was then fed one-half of its daily ration. After sufficient time for gastric emptying had elapsed (4 to 5 hours), Histalog was again injected, and the stomach was drained for another 2 -hour period. The fluid from both periods was then pooled, and the sodium, potassium, and chloride concentrations were determined. The amount of sodium and potassium removed in the gastric juice was returned to the animal by adding an equivalent amount of sodium and potassium chloride to the evening feeding. The diet was also supplemented by a volume of distilled water equal to the volume of gastric juice removed during drainage.

Each animal was fed $30 \mathrm{~g}$ per $\mathrm{kg}$ of an artificial diet (homogenized with 1.5 times its weight of distilled water), the composition of which has been described previously (1). The intrinsic electrolyte content of the diet was sodium, $1 \mathrm{mEq}$ per $100 \mathrm{~g}$; potassium, less than 0.1 $\mathrm{mEq}$ per $100 \mathrm{~g}$; and chloride, $1 \mathrm{mEq}$ per $100 \mathrm{~g}$. The diet was supplemented by 2 mmoles per $\mathrm{kg}$ of neutral phosphate (4 $\left.\mathrm{HPO}_{4} / 1 \quad \mathrm{H}_{2} \mathrm{PO}_{4}\right)$, administered as 2.4 $\mathrm{mEq}$ per $\mathrm{kg}$ of the potassium and $1.2 \mathrm{mEq}$ per $\mathrm{kg}$ of the sodium salt. The cation composition of the phosphate supplement was varied during the latter part of the study (vide infra).

Each study was initiated by a control period of 6 to 9 days. Detailed balance observations were made over the last 4 or 5 control days. The study was then divided into four periods.

Period I: gastric drainage. Gastric drainage was carried out, as described above, until 5 to $7 \mathrm{mEq}$ of hydrochloric acid per $\mathrm{kg}$ of body weight had been removed. A 2- to 4-day period was required for this purpose.

Period II. Balance observations were continued for 6 to 10 days after the gastric drainage had been discontinued.

1 The authors wish to express their appreciation to Drs. Donald J. Glotzer, George L. McDonald, and Barry F. Sachs for performing the surgical procedures.

2 Eli Lilly, Indianapolis, Ind. 
Period III: correction phase. Attempts to correct the alkalosis were made according to three protocols:

A) Sodium phosphate supplement (potassium-free and chloride-free intake). The electrolyte supplement of two animals ( $Z$ and $E$ ) was modified by replacing the 2.4 $\mathrm{mEq}$ of potassium phosphate by an equivalent amount of sodium phosphate. The total sodium intake was thus increased to $3.6 \mathrm{mEq}$ per $\mathrm{kg}$ without changing the anion content of the diet. Balance observations were continued for 5 days.

B) Sodium chloride supplement (potassium-free intake). The electrolyte supplement of five animals was modified by replacing the $2.4 \mathrm{mEq}$ per $\mathrm{kg}$ of potassium phosphate by an equivalent amount of sodium phosphate. In addition, they were given 5 mmoles per $\mathrm{kg}$ of sodium chloride. [One of the animals had been given a sodium phosphate supplement (Protocol A) immediately before being given sodium chloride.] Balance observations were continued for 5 to 7 days.

C) Potassium chloride supplement (sodium-free intake). The electrolyte supplement of two animals (E and C-2) was modified by replacing the sodium phosphate by an equivalent amount of potassium phosphate. In addition, the animals were given $5 \mathrm{mmoles}$ per $\mathrm{kg}$ of potassium chloride. [One animal had been given a sodium phosphate supplement (Protocol A) immediately before being given potassium chloride.] Balance observations were continued for 4 days.

Period IV: sodium bicarbonate administration. Four animals that had been subjected to Protocol B (i.e., sodium chloride supplement during administration of a potassium-free intake) were given a sodium bicarbonate supplement of 5 mmoles per $\mathrm{kg}$ for 4 to 6 days in order to assess the renal reabsorption of bicarbonate at the height of the potassium deficiency. In three of the four animals the sodium phosphate intake was simultaneously reduced or discontinued. One animal received $30 \mathrm{mEq}$ of sodium chloride per day throughout the period of bicarbonate feeding.

Creatinine clearance studies. Alkalosis was induced in four additional animals by the gastric drainage technique described above. Each dog underwent exogenous creatinine clearance studies on 3 days during the control period and further clearance studies on the last 3 days of Period II. Clearances were performed a minimum of 16 hours after the last feeding. An adequate urine flow was assured by the infusion of $5 \%$ mannitol solution at a rate of approximately $2 \mathrm{ml}$ per minute.

TABLE I

Changes in electrolyte balance during and after gastric drainage

\begin{tabular}{|c|c|c|c|c|c|c|c|c|c|c|c|c|c|}
\hline \multirow[b]{3}{*}{ Period } & \multirow[b]{3}{*}{ Dog } & \multirow[b]{3}{*}{ Days } & \multirow{2}{*}{\multicolumn{2}{|c|}{ Weight }} & \multicolumn{6}{|c|}{ External balance } & \multicolumn{3}{|c|}{ Internal balance } \\
\hline & & & & & \multirow{2}{*}{$\mathrm{Cl}$} & \multirow{2}{*}{$\mathrm{Na}$} & \multirow{2}{*}{$\mathrm{Cl}-\left(\frac{\mathrm{Na}}{1.3}\right)$} & \multirow{2}{*}{$\mathbf{K}$} & \multirow{2}{*}{$\mathrm{Kn} *$} & \multirow{2}{*}{$\mathrm{N}$} & \multirow{2}{*}{$\begin{array}{l}\text { Intra- } \\
\text { cellular } \\
\mathbf{K}\end{array}$} & \multirow{2}{*}{$\begin{array}{l}\text { Intra- } \\
\text { cellular } \\
\mathrm{Na}\end{array}$} & \multirow{2}{*}{$\begin{array}{l}\text { ECF† } \\
\text { volume }\end{array}$} \\
\hline & & & Initial & Final & & & & & & & & & \\
\hline & & & $k g$ & $\mathrm{~kg}$ & $m E q$ & $m E q$ & $m E q$ & $m E q$ & $m E q$ & $g$ & $m E q$ & $m E q$ & $L$ \\
\hline \multicolumn{14}{|c|}{ I. Gastric drainage } \\
\hline & K & 4 & 12.7 & 12.2 & -68 & -26 & -17 & -39 & -52 & +5.1 & -48 & +7 & -0.4 \\
\hline & $Z$ & 3 & 14.4 & 14.3 & -87 & -20 & -72 & -26 & -35 & +3.3 & -30 & +23 & -0.2 \\
\hline & $\mathrm{D}$ & 4 & 14.2 & 14.1 & -81 & -46 & -46 & -23 & -40 & +6.4 & -37 & +20 & -0.3 \\
\hline & $\mathrm{E}$ & 4 & 13.6 & 13.5 & -87 & -6 & -82 & -25 & -31 & +2.2 & -27 & +91 & -0.5 \\
\hline & $\mathbf{O}$ & 2 & 14.3 & 14.4 & -73 & -38 & -44 & -29 & -26 & -1.1 & -23 & +13 & -0.4 \\
\hline & C-1 & 4 & 12.2 & 11.6 & -90 & -72 & -35 & -60 & -62 & +0.5 & -59 & o & -0.4 \\
\hline & $\mathrm{C}-2$ & 4 & 13.2 & 12.9 & -70 & -42 & -38 & -27 & -40 & +4.8 & -36 & +10 & -0.3 \\
\hline \multirow[t]{7}{*}{ II. } & $\mathrm{K}$ & 8 & 12.2 & 12.1 & +4 & +30 & -17 & -27 & -33 & +2.1 & -31 & +22 & +0.2 \\
\hline & $Z$ & 6 & 14.3 & 14.1 & +14 & +23 & -4 & -16 & -17 & +0.3 & -20 & +15 & +0.1 \\
\hline & $D \ddagger$ & 8 & 14.1 & 1.3 .9 & -6 & +11 & -14 & -1 & -26 & +9.1 & -27 & -19 & o \\
\hline & $\mathrm{E}$ & 6 & 13.5 & 13.5 & +14 & +24 & -4 & -7 & -9 & +0.8 & -11 & -36 & +0.2 \\
\hline & $\mathrm{O}$ & 7 & 14.4 & 14.0 & +20 & o & +20 & -12 & +33 & -16.8 & +32 & -24 & +0.3 \\
\hline & C-1 & 10 & 11.6 & 11.6 & +13 & +19 & -2 & -25 & -8 & -5.9 & -9 & +29 & +0.2 \\
\hline & $\mathrm{C}-2$ & 6 & 12.9 & 12.8 & +8 & +7 & +3 & -23 & -28 & +2.0 & -29 & -10 & +0.2 \\
\hline \multicolumn{14}{|c|}{ III. A. Sodium phosphate supplement } \\
\hline & $\mathbf{Z}$ & 5 & 14.1 & 14.1 & -1 & +26 & -20 & -87 & -85 & -0.5 & -81 & +34 & +0.1 \\
\hline & $\mathrm{E}$ & 5 & 13.5 & 13.5 & +10 & +36 & -18 & -71 & -79 & +3.0 & -77 & +6 & +0.2 \\
\hline \multicolumn{14}{|c|}{ B. Sodium chloride supplement } \\
\hline & $\mathbf{K}$ & 5 & 12.1 & 12.3 & +152 & +65 & +100 & -35 & -37 & +0.3 & -39 & -20 & +0.8 \\
\hline & $Z$ & 7 & 14.1 & 14.4 & +192 & +83 & +125 & -18 & -34 & +5.5 & -38 & -58 & +1.1 \\
\hline & $\mathrm{D}$ & 5 & 13.9 & 14.3 & +163 & +83 & +99 & -25 & -48 & +8.5 & -50 & -18 & +0.7 \\
\hline & $\mathrm{O}$ & 5 & 14.0 & 14.1 & +217 & +159 & +95 & -43 & -24 & -5.4 & -27 & -27 & +1.1 \\
\hline & C-1 & 7 & 11.6 & 12.2 & +240 & +200 & +85 & -44 & -55 & +4.2 & -57 & -12 & +1.2 \\
\hline \multicolumn{14}{|c|}{ C. Potassium chloride supplement } \\
\hline & E & 4 & 13.5 & 13.5 & +239 & -44 & +273 & +173 & +172 & -0.1 & +159 & +236 & +1.3 \\
\hline & C-2 & 4 & 12.8 & 12.8 & +116 & +2 & +114 & +94 & +84 & +3.2 & +75 & -89 & +0.5 \\
\hline
\end{tabular}

$* \mathrm{Kn}=\mathrm{K}$ corrected for $\mathrm{N}$.

$\ddagger \mathrm{ECF}=$ extracellular fluid.
$\ddagger$ Vomited on day 3 ; electrolyte content included in output. 


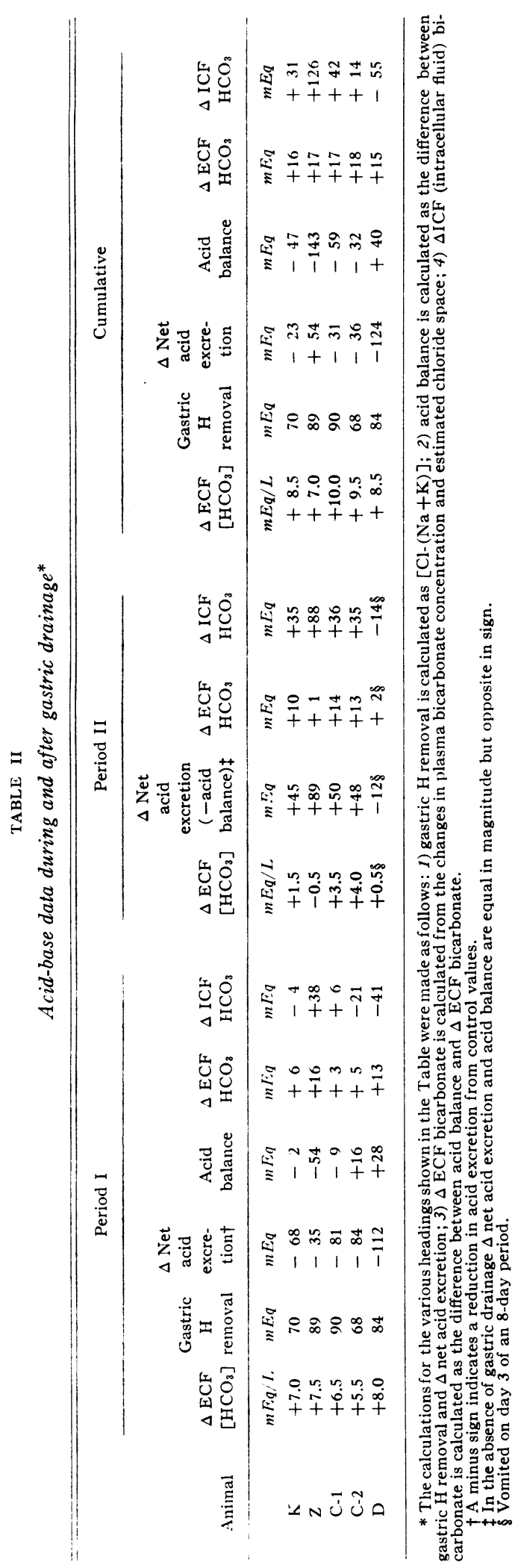

The analytic methods and technique of balance have been described previously $(2,3)$ except that $\mathrm{pH}$ was determined by means of an Epsco blood parameter analyzer. The total acid removed in the gastric juice was taken to be $[\mathrm{Cl}-(\mathrm{Na}+\mathrm{K})]$. This estimated value was found to agree closely with the values obtained by titration with sodium hydroxide. Internal exchanges of sodium, potassium, and hydrogen were estimated in the usual fashion from chloride space calculations.

\section{Results}

Observations on plasma composition and electrolyte balance (Table I) were made in seven studies. Satisfactory data on urinary acid excretion could be obtained in only five studies (Table II) ; in one of the remaining studies there was a urinary tract infection with a urea splitting organism $(\operatorname{dog} \mathrm{E})$, and in the other there were wide fluctuations in acid excretion during the control period $(\operatorname{dog} \mathrm{O})$. Changes in plasma composition and electrolyte balance for a representative study are shown in Figure 1 , and detailed balance data for two studies are shown in Tables III and IV.

At the end of the control period, average plasma concentrations were as follows : sodium, $147 \mathrm{mEq}$ per L; chloride, $109 \mathrm{mEq}$ per L; bicarbonate, 22.5 $\mathrm{mEq}$ per L; and potassium, $4.1 \mathrm{mEq}$ per L. Arterial blood $\mathrm{pH}$ varied from 7.41 to 7.49 .

\section{Period I: gastric drainage}

1) Acid-base balance (Table II). Plasma bicarbonate concentration rose by an average of 7.0 $\mathrm{mEq}$ per $\mathrm{L}$ to a final mean level of $29.5 \mathrm{mEq}$ per L. Blood $\mathrm{pH}$ rose to a range of 7.50 to 7.55 . The amount of acid removed by gastric drainage averaged $81 \mathrm{mEq}$ (range, 68 to $90 \mathrm{mEq}$ ). Renal acid excretion $\left[\mathrm{NH}_{4}{ }^{+}+\right.$titratable acid $\left.-\mathrm{HCO}_{3}\right]$ was suppressed by an average of $76 \mathrm{mEq}$. The relationship between acid removal and acid excretion is shown for a representative study in Figure 2.

2) Chloride, potassium, and sodium (Table I). Plasma chloride concentration fell by $13 \mathrm{mEq}$ per $\mathrm{L}$, to a final average value of $96 \mathrm{mEq}$ per $\mathrm{L}$. The net chloride deficit was $79 \mathrm{mEq}$ (range, 68 to $90 \mathrm{mEq}$ ). Plasma sodium concentration was virtually unchanged, but plasma potassium concentration fell by $1.1 \mathrm{mEq}$ per $\mathrm{L}$ to an average of $3.0 \mathrm{mEq}$ per L. Potassium losses (corrected for $\mathrm{N}$ ) averaged $41 \mathrm{mEq}$ (range, 26 to $62 \mathrm{mEq}$ ), and 


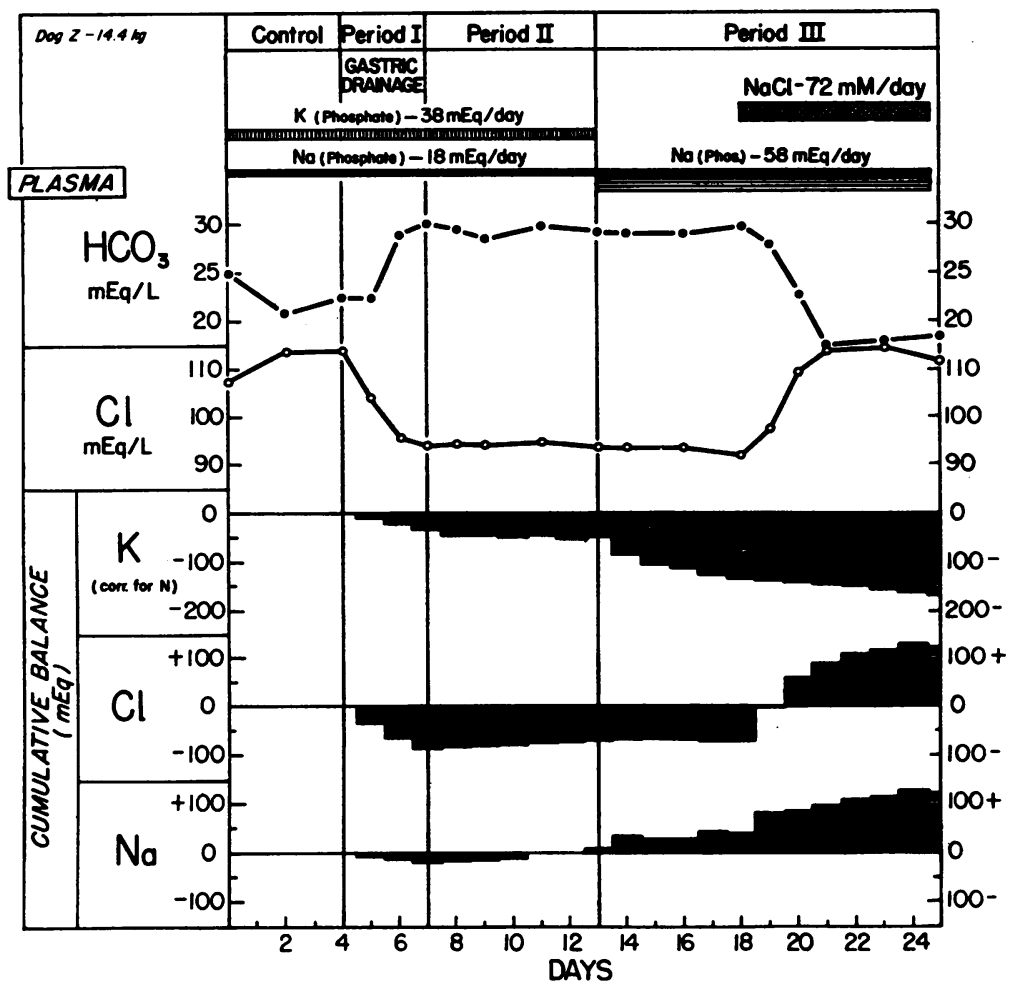

Fig. 1. Plasma composition and electrolyte balance in a RePresentaTIVE STUDY OF SELECTIVE HCL DEPLETION ( $\operatorname{dog} Z$ ).

sodium loss averaged $36 \mathrm{mEq}$ (range, 6 to 72 $\mathrm{mEq}$ ). Calculated extracellular fluid volume fell by an average of $0.4 \mathrm{~L}$.

\section{Period II}

1) Acid-base balance (Table II). In all but one of the seven studies plasma bicarbonate rose slightly (average, $2.0 \mathrm{mEq}$ per $\mathrm{L}$ ). At the end of the period average plasma bicarbonate concentration was $31.5 \mathrm{mEq}$ per $\mathrm{L}$, a rise of $9.0 \mathrm{mEq}$ per $\mathrm{L}$ from control. Blood $\mathrm{pH}$ ranged from 7.51 to 7.59. In the four studies in which net acid excretion rose, the average increment above control was $58 \mathrm{mEq}$. This rise was due almost entirely to a fall in urine $\mathrm{pH}$ and an increase in excretion of titratable acid. In one study ( $\operatorname{dog} \mathrm{D}$, which vomited on day 3 ) acid excretion during the period did not differ significantly from control. Cumulative acid balance for Periods I and II is shown in Table II.

2) Chloride, potassium, and sodium (Table I). There was no significant change in plasma chlo-
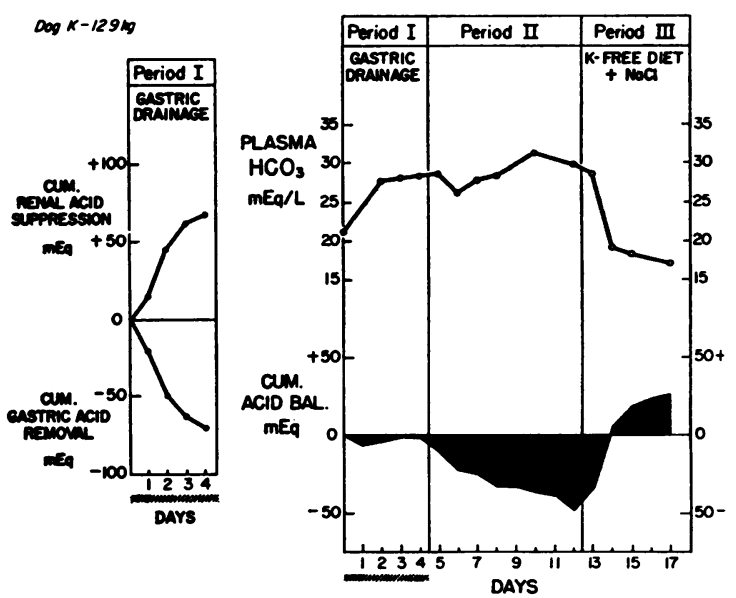

Fig. 2. Gastric acid REMoval, Change IN URINARY NET ACID EXCRETION, AND CUMULATIVE ACID BALANCE DURING DEVELOPMENT AND CORRECTION OF METABOLIC ALKALosis $(\operatorname{dog} \mathrm{K})$. Cumulative acid balance for Period I is calculated as the algebraic sum of gastric acid removal and suppression of net acid. Subsequent changes in acid balance are solely the result of changes in acid excretion. 
TABLE III Balance data on dog subjected to chloride

\begin{tabular}{|c|c|c|c|c|c|c|c|c|c|c|c|c|c|}
\hline & \multirow[b]{2}{*}{ Day } & \multirow{2}{*}{$\begin{array}{l}\text { Chloride } \\
\text { removed } \\
\text { from } \\
\text { stomach* }\end{array}$} & \multirow{2}{*}{$\begin{array}{c}\text { Body } \\
\text { wt }\end{array}$} & \multicolumn{4}{|c|}{ Intake } & \multicolumn{6}{|c|}{ Urine } \\
\hline & & & & $\mathrm{Na}$ & $\mathrm{Cl}$ & $\mathbf{K}$ & $\mathrm{N}$ & Vol & $\mathrm{pH}$ & $\mathrm{HCO}_{3}$ & $\mathrm{Cl}$ & $\mathrm{Na}$ & $\mathrm{K}$ \\
\hline & & $m E q$ & $k g$ & $m E q$ & $m E q$ & $m E \boldsymbol{q}$ & $g$ & $m l$ & & $m E q$ & $m E q$ & $m E q$ & $m E q$ \\
\hline \multirow{4}{*}{$\begin{array}{l}\text { Control } \\
\text { period }\end{array}$} & 1 & & 14.5 & 23 & 5 & 38 & 8.2 & 560 & 6.31 & 4 & 2 & 15 & 32 \\
\hline & 2 & & 14.4 & 22 & 4 & 37 & 8.0 & 500 & 6.12 & 1 & 2 & 9 & 30 \\
\hline & 3 & & 14.5 & 23 & 5 & 38 & 8.2 & 410 & 6.12 & 2 & 2 & 19 & 42 \\
\hline & 4 & & 14.5 & 23 & 5 & 38 & 8.2 & 500 & 6.27 & 3 & 2 & 25 & 42 \\
\hline \multirow{3}{*}{$\begin{array}{l}\text { Period I } \\
\text { Gastric } \\
\text { drainage }\end{array}$} & 5 & 36 & 14.4 & 22 & 4 & 37 & 8.0 & 220 & 6.53 & 3 & 2 & 24 & 40 \\
\hline & 6 & 29 & 14.4 & 23 & 5 & 38 & 8.2 & 450 & 6.70 & 12 & 2 & 22 & 49 \\
\hline & 7 & 23 & 14.4 & 23 & 5 & 38 & 8.2 & 430 & 6.41 & 6 & 0 & 24 & 47 \\
\hline \multirow[t]{6}{*}{ Period II } & 8 & & 14.3 & 23 & 5 & 36 & 8.2 & 560 & 5.91 & 1 & 0 & 17 & 46 \\
\hline & 9 & & 14.2 & 23 & 5 & 36 & 8.2 & 325 & 5.58 & 1 & 0 & 14 & 33 \\
\hline & 10 & & 14.2 & 23 & 5 & 36 & 8.2 & 400 & 5.75 & 1 & 0 & 12 & 42 \\
\hline & 11 & & 14.1 & 23 & 5 & 36 & 8.2 & 420 & 5.67 & 1 & 0 & 12 & 32 \\
\hline & 12 & & 14.1 & 23 & 5 & 36 & 8.2 & 410 & 5.89 & 1 & 1 & 16 & 41 \\
\hline & 13 & & 14.1 & 23 & 5 & 36 & 8.2 & 510 & 5.88 & 1 & 1 & 14 & 32 \\
\hline \multirow{5}{*}{$\begin{array}{l}\text { Period IIIa } \\
\text { Sodium } \\
\text { phosphate } \\
\text { supplement }\end{array}$} & 14 & & 14.1 & 58 & 5 & 0 & 8.2 & 250 & 5.96 & 1 & 1 & 19 & 34 \\
\hline & 15 & & 14.2 & 58 & 5 & 0 & 8.2 & 450 & 6.26 & 5 & 1 & 55 & 25 \\
\hline & 16 & & 14.1 & 57 & 4 & 0 & 8.1 & 440 & 6.20 & 3 & 3 & 48 & 6 \\
\hline & 17 & & 14.0 & 57 & 4 & 0 & 8.0 & 290 & 6.24 & 4 & 2 & 30 & 9 \\
\hline & 18 & & 14.1 & 54 & 4 & 0 & 8.0 & 635 & 6.42 & 5 & 3 & 52 & 8 \\
\hline \multirow{7}{*}{$\begin{array}{l}\text { Period IIIb } \\
\text { Sodium } \\
\text { chloride } \\
\text { supplement }\end{array}$} & 19 & & 14.1 & 124 & 73 & 0 & 8.0 & 380 & 6.60 & 4 & 3 & 71 & 4 \\
\hline & 20 & & 14.3 & 127 & 76 & 0 & 8.1 & 640 & 7.49 & 25 & 12 & 114 & 1 \\
\hline & 21 & & 14.4 & 127 & 76 & 0 & 8.1 & 580 & 6.83 & 11 & 43 & 108 & 2 \\
\hline & 22 & & 14.4 & 127 & 76 & 0 & 8.1 & 690 & 6.58 & 5 & 53 & 105 & 1 \\
\hline & 23 & & 14.3 & 124 & 74 & 0 & 8.0 & 810 & 6.68 & 7 & 65 & 113 & 1 \\
\hline & 24 & & 14.3 & 124 & 74 & 0 & 8.0 & 600 & 6.63 & 3 & 56 & 101 & 1 \\
\hline & 25 & & 14.4 & 128 & 77 & 0 & 8.2 & 830 & 6.57 & 5 & 85 & 129 & 1 \\
\hline
\end{tabular}

* The $\mathrm{Na}$ and $\mathrm{K}$ removed in the gastric juice were replaced by administration of an equivalent amount of $\mathrm{NaCl}$ and $\mathrm{KCl}$. Thus, "chloride removed from stomach" refers to that chloride withdrawn as $\mathrm{HCl}$ and not replaced.

† $\mathrm{TA}=$ titratable acid.

TABLE IV

Balance data on dog subjected to chloride

\begin{tabular}{|c|c|c|c|c|c|c|c|c|c|c|c|c|c|}
\hline & \multirow{2}{*}{ Day } & \multirow{2}{*}{$\begin{array}{l}\text { Chloride } \\
\text { removed } \\
\text { from } \\
\text { stomach* }\end{array}$} & \multirow{2}{*}{$\begin{array}{c}\text { Body } \\
\text { wt }\end{array}$} & \multicolumn{4}{|c|}{ Intake } & \multicolumn{6}{|c|}{ Urine } \\
\hline & & & & $\mathrm{Na}$ & $\mathrm{Cl}$ & $\mathrm{K}$ & $\mathbf{N}$ & Vol & $\mathrm{pH}$ & $\mathrm{HCO}_{3}$ & $\mathrm{Cl}$ & $\mathrm{K}$ & $\mathrm{Na}$ \\
\hline & & $m E q$ & $k g$ & $m E q$ & $m E q$ & $m E q$ & $g$ & $m l$ & & $m E q$ & $m E q$ & $m E q$ & $m E q$ \\
\hline \multirow{4}{*}{$\begin{array}{l}\text { Control } \\
\text { period }\end{array}$} & 1 & & 12.5 & 17 & 4 & 29 & 7.0 & 740 & 6.71 & 6 & 3 & 26 & 11 \\
\hline & 2 & & 12.5 & 17 & 4 & 29 & 7.1 & 605 & 6.35 & 2 & 2 & 30 & 8 \\
\hline & 3 & & 12.5 & 17 & 4 & 29 & 7.1 & 540 & 6.60 & 7 & 1 & 29 & 7 \\
\hline & 4 & & 12.7 & 17 & 4 & 29 & 7.1 & 690 & 6.58 & 4 & 2 & 28 & 14 \\
\hline \multirow{4}{*}{$\begin{array}{l}\text { Period I } \\
\text { Gastric } \\
\text { drainage }\end{array}$} & 5 & 21 & & 17 & 4 & 29 & 7.1 & 600 & 6.48 & 4 & 1 & 34 & 16 \\
\hline & 6 & 28 & 12.4 & 17 & 4 & 29 & 7.0 & 720 & 6.75 & 9 & 1 & 38 & 25 \\
\hline & 7 & 14 & 12.3 & 17 & 4 & 29 & 7.0 & 710 & 6.60 & 8 & 1 & 41 & 13 \\
\hline & 8 & 7 & 12.2 & 17 & 4 & 28 & 6.8 & 620 & 6.19 & 2 & 1 & 37 & 7 \\
\hline \multirow[t]{8}{*}{ Period II } & 9 & & 12.1 & 17 & 4 & 28 & 6.8 & 640 & 6.22 & 3 & 1 & 32 & 9 \\
\hline & 10 & & 12.1 & 17 & 4 & 28 & 6.9 & 850 & 6.19 & 3 & 1 & 29 & 4 \\
\hline & 11 & & 12.0 & 17 & 4 & 28 & 6.8 & 610 & 6.24 & 3 & 1 & 35 & 7 \\
\hline & 12 & & 12.0 & 17 & 4 & 28 & 6.9 & 540 & 5.97 & 1 & 1 & 25 & 1 \\
\hline & 13 & & 11.9 & 16 & 4 & 28 & 6.7 & 460 & 6.02 & 1 & 1 & 28 & 3 \\
\hline & 14 & & 12.1 & 17 & 4 & 28 & 6.8 & 340 & 6.29 & 2 & 1 & 30 & 3 \\
\hline & 15 & & 12.1 & 17 & 4 & 29 & 7.0 & 470 & 6.20 & 2 & 1 & 32 & 4 \\
\hline & 16 & & 12.1 & 17 & 4 & 28 & 6.9 & 590 & 6.58 & 5 & 1 & 33 & 8 \\
\hline Period III & 17 & & 12.3 & 109 & 67 & 0 & 6.9 & 350 & 6.92 & 14 & 1 & 26 & 31 \\
\hline Sodium & 18 & & 12.2 & 112 & 69 & $\mathbf{0}$ & 7.1 & 620 & 8.03 & 39 & 11 & 1 & 103 \\
\hline chloride & 19 & & 12.2 & 112 & 69 & $\mathbf{0}$ & 7.1 & 540 & 7.34 & 16 & 43 & 1 & 93 \\
\hline \multirow[t]{2}{*}{ supplement } & 20 & & 12.2 & 112 & 69 & 0 & 7.1 & 440 & 6.71 & 4 & 60 & 1 & 94 \\
\hline & 21 & & 12.3 & 112 & 69 & $\mathbf{0}$ & 7.1 & 510 & 6.78 & 7 & 63 & 1 & , 99 \\
\hline
\end{tabular}

* The $\mathrm{Na}$ and $\mathrm{K}$ removed in the gastric juice were replaced by administration of an equivalent amount of $\mathrm{NaCl}$ and $\mathrm{KCl}$. Thus, "chloride removed from stomach" refers to that chloride withdrawn as $\mathrm{HCl}$ and not replaced.

† TA = titratable acidity. 
TABLE III

depletion by gastric drainage $(\operatorname{dog} Z)$

\begin{tabular}{|c|c|c|c|c|c|c|c|c|c|c|c|c|c|c|}
\hline \multicolumn{5}{|c|}{ Lrine } & \multicolumn{4}{|c|}{ Stool } & \multicolumn{6}{|c|}{ Plasma } \\
\hline $\mathrm{NH}_{4}$ & TA $†$ & $\begin{array}{c}\text { Organic } \\
\text { acids }\end{array}$ & $\mathrm{PO}_{4}$ & $\mathrm{~N}$ & $\mathrm{Na}$ & $\mathrm{Cl}$ & $\mathbf{K}$ & $\mathbf{N}$ & $\mathrm{PcO}_{2}$ & $\mathrm{pH}$ & $\mathrm{HCO}_{3}$ & $\mathrm{Na}$ & $\mathrm{Cl}$ & $\mathbf{K}$ \\
\hline$m E q$ & $m E q$ & $m E q$ & mmoles & $g$ & $m E q$ & $m E_{q}$ & $m E q$ & $\boldsymbol{g}$ & $m m H g$ & & $m E q / L$ & $m E q / L$ & $m E q / L$ & $m E q / L$ \\
\hline 27 & 23 & 13 & 40 & 5.5 & 5 & 1 & 1 & 0.8 & & & & & & \\
\hline 22 & 21 & 12 & 33 & 5.9 & 5 & 1 & 1 & 0.8 & 30 & 7.46 & 20.9 & 148 & 113 & 4.2 \\
\hline 29 & 31 & 22 & 48 & 7.9 & 5 & 1 & 1 & 0.8 & & & & & & \\
\hline 33 & 30 & 23 & 50 & 8.7 & 5 & 1 & 1 & 0.8 & 33 & 7.45 & 22.3 & 149 & 114 & 4.3 \\
\hline 16 & 17 & 17 & 37 & 5.8 & 3 & 1 & 1 & 0.4 & 32 & 7.46 & 22.2 & 147 & 104 & 4.2 \\
\hline 43 & 18 & 21 & 46 & 7.2 & 3 & 1 & 1 & 0.4 & 38 & 7.50 & 28.8 & 147 & 96 & 3.8 \\
\hline 23 & 24 & 21 & 45 & 6.9 & 3 & 1 & 1 & 0.4 & 39 & 7.51 & 30.0 & 145 & 94 & 2.8 \\
\hline 34 & 40 & 23 & 55 & 8.5 & 3 & 1 & 1 & 0.4 & 38 & 7.51 & 29.4 & 146 & 96 & 3.0 \\
\hline 22 & 29 & 14 & 37 & 6.3 & 3 & 1 & 1 & 0.4 & 37 & 7.51 & 28.5 & 145 & 94 & 3.3 \\
\hline 43 & 40 & 25 & 53 & 9.0 & 3 & 1 & 1 & 0.4 & & & & & & \\
\hline 32 & 33 & 19 & 42 & 7.4 & 3 & 1 & 1 & 0.4 & 36 & 7.54 & 29.6 & 147 & 95 & 3.0 \\
\hline 34 & 34 & 21 & 47 & 8.2 & 3 & 1 & 1 & 0.4 & & & & & & \\
\hline 31 & 29 & 17 & 41 & 7.1 & 3 & 1 & 1 & 0.4 & 38 & 7.51 & 29.2 & 143 & 94 & 3.7 \\
\hline 24 & 29 & 18 & 40 & 6.5 & 9 & 1 & 1 & 1.0 & 37 & 7.51 & 28.9 & 146 & 94 & 2.5 \\
\hline 41 & 34 & 27 & 56 & 9.6 & 9 & 1 & 1 & 1.0 & & & & & & \\
\hline 32 & 26 & 17 & 40 & 7.0 & 9 & 1 & 1 & 1.0 & 38 & 7.51 & 29.0 & 145 & 94 & 2.5 \\
\hline 33 & 20 & 16 & 32 & 5.7 & 9 & 1 & 1 & 1.0 & & & & & & \\
\hline 38 & 24 & 21 & 44 & 7.2 & 9 & 1 & 1 & 1.0 & 36 & 7.54 & 29.7 & 143 & 92 & 2.1 \\
\hline 35 & 21 & 21 & 48 & 8.2 & 6 & 1 & 1 & 0.7 & 39 & 7.47 & 27.8 & 145 & 97 & 2.6 \\
\hline 25 & $\mathbf{0}$ & 22 & 40 & 6.7 & 6 & 1 & 1 & 0.7 & 39 & 7.39 & 22.7 & 147 & 109 & 2.9 \\
\hline 28 & 11 & 17 & 38 & 6.7 & 6 & 1 & 1 & 0.7 & 28 & 7.41 & 17.4 & 147 & 115 & 2.7 \\
\hline 35 & 16 & 19 & 38 & 6.6 & 6 & 1 & 1 & 0.7 & & & & & & \\
\hline 36 & 13 & 15 & 36 & 6.0 & 6 & 1 & 1 & 0.7 & 29 & 7.41 & 17.8 & 146 & 115 & 2.5 \\
\hline 31 & 14 & 14 & 36 & 5.9 & 6 & 1 & 1 & 0.7 & & & & & & \\
\hline 38 & 17 & 17 & 39 & 6.0 & 6 & 1 & 1 & 0.7 & 30 & 7.41 & 18.2 & 146 & 112 & 2.5 \\
\hline
\end{tabular}

TABLE IV

depletion by gastric drainage (dog $K)$

\begin{tabular}{|c|c|c|c|c|c|c|c|c|c|c|c|c|c|c|}
\hline \multicolumn{5}{|c|}{ Urine } & \multicolumn{4}{|c|}{ Stool } & \multicolumn{5}{|c|}{ Plasma } & \multirow[b]{2}{*}{$\mathbf{K}$} \\
\hline $\mathrm{NH}_{4}$ & TAt & acids & $\mathrm{PO}_{4}$ & $N$ & $\mathrm{Na}$ & $\mathrm{Cl}$ & $\mathbf{K}$ & $N$ & $\mathrm{PcO}_{2}$ & $\mathrm{pH}$ & $\mathrm{HCO}_{3}$ & $\mathrm{Na}$ & $\mathrm{Cl}$ & \\
\hline$m E q$ & $m E q$ & $m E q$ & mmoles & $g$ & $m E q$ & $m E q$ & $m E q$ & $g$ & $m m H g$ & & $m E q / L$ & $m E q / L$ & $m E q / L$ & $m E q_{\mathbf{q}} / L$ \\
\hline 31 & 11 & 14 & 29 & 4.4 & 4 & 1 & 1 & 0.5 & & & & & & \\
\hline 32 & 19 & 15 & 34 & 5.4 & 4 & 1 & 1 & 0.5 & 37 & 7.42 & 23.4 & 146 & 110 & 4.1 \\
\hline 46 & 15 & 15 & 35 & 5.4 & 4 & 1 & 1 & 0.5 & & & & & & \\
\hline 32 & 15 & 15 & 34 & 6.1 & 4 & 1 & 1 & 0.5 & 30 & 7.47 & 21.1 & 144 & 109 & 4.3 \\
\hline 19 & 16 & 13 & 32 & 4.9 & 6 & 1 & 1 & 0.6 & & & & & & \\
\hline 14 & 12 & 14 & 33 & 4.6 & 6 & 1 & 1 & 0.6 & 36 & 7.51 & 27.8 & 151 & 106 & 4.1 \\
\hline 20 & 16 & 15 & 35 & 5.5 & 6 & 1 & 1 & 0.6 & 36 & 7.52 & 28.1 & 148 & & 3.8 \\
\hline 20 & 22 & 18 & 34 & 5.4 & 6 & 1 & 1 & 0.6 & 37 & 7.50 & 28.3 & 148 & 100 & 3.0 \\
\hline 34 & 22 & 15 & 34 & 6.4 & 6 & 1 & 1 & 0.6 & 35 & 7.54 & 28.6 & 148 & 99 & 3.0 \\
\hline 38 & 23 & 24 & 36 & 6.5 & 6 & 1 & 1 & 0.6 & 34 & 7.51 & 26.3 & 148 & 97 & 3.4 \\
\hline 30 & 21 & 15 & 33 & 6.3 & 6 & 1 & 1 & 0.6 & 36 & 7.51 & 27.9 & 146 & 102 & 2.9 \\
\hline 31 & 24 & 15 & 33 & 5.9 & 6 & 1 & 1 & 0.6 & 34 & 7.54 & 28.4 & 144 & 98 & 3.2 \\
\hline 25 & 22 & 12 & 31 & 5.3 & 6 & 1 & 1 & 0.6 & & & & & & \\
\hline 31 & 21 & 15 & 34 & 6.4 & 6 & 1 & 1 & 0.6 & 37 & 7.55 & 31.3 & 142 & 94 & 2.9 \\
\hline 27 & 22 & 13 & 34 & 5.3 & 6 & 1 & 1 & 0.6 & & & & & & \\
\hline 41 & 17 & 13 & 37 & 5.8 & 6 & 1 & 1 & 0.6 & 35 & 7.55 & 29.8 & 144 & 94 & 3.0 \\
\hline 37 & 9 & 18.4 & 31 & 6.6 & 12 & 1 & 1 & 1.2 & 35 & 7.53 & 28.6 & 144 & 101 & 2.4 \\
\hline 47 & 0 & 0 & 35 & 5.3 & 12 & 1 & 1 & 1.2 & 30 & 7.43 & 19.0 & 147 & 112 & 3.1 \\
\hline 49 & 1 & 15 & 32 & 6.0 & 12 & 1 & 1 & 1.2 & 28 & 7.43 & 18.1 & 148 & 116 & 3.4 \\
\hline 34 & 11 & 15 & 31 & 5.9 & 12 & 1 & 1 & 1.2 & & & & & & 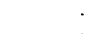 \\
\hline 40 & 10 & 13 & 32 & 5.2 & 12 & 1 & 1 & 1.2 & 28 & 7.40 & 17.0 & 148 & 114 & 2.9 \\
\hline
\end{tabular}


ride concentration, and the urine remained virtually chloride free. Plasma potassium and sodium concentrations varied little from the preceding period. Potassium balance (corrected for $\mathrm{N}$ ) during Period II was slightly negative in all but one dog. Sodium balance for the period was slightly positive. Calculated extracellular volume rose by an average of $0.2 \mathrm{~L}$.

Average cumulative balance values for Periods I and II were as follows: potassium (corrected for $\mathrm{N}$ ), $-53 \mathrm{mEq}$ (range, +7 to -83 ), and sodium $-20 \mathrm{mEq}$ (range, +18 to -53 ). Extracellular fluid volume fell by an average of $0.2 \mathrm{~L}$.

\section{Period III : correction phase}

Protocol A. Sodium phosphate (potassium-free and chloride-free intake). Administration of sodium phosphate induced no change in acid-base balance or in plasma chloride values. Plasma potassium concentration fell by $1.3 \mathrm{mEq}$ per $\mathrm{L}$ to an average of $2.2 \mathrm{mEq}$ per $\mathrm{L}$, and there was a further negative potassium balance (corrected for $\mathrm{N}$ ) of -79 and $-85 \mathrm{mEq}$ per L. Extracellular volume rose by 0.1 and $0.2 \mathrm{~L}$.

Protocol B. Sodium chloride (potassium-free intake) and Protocol C. Potassium chloride (sodium-free intake). 1) Acid-base balance. Plasma

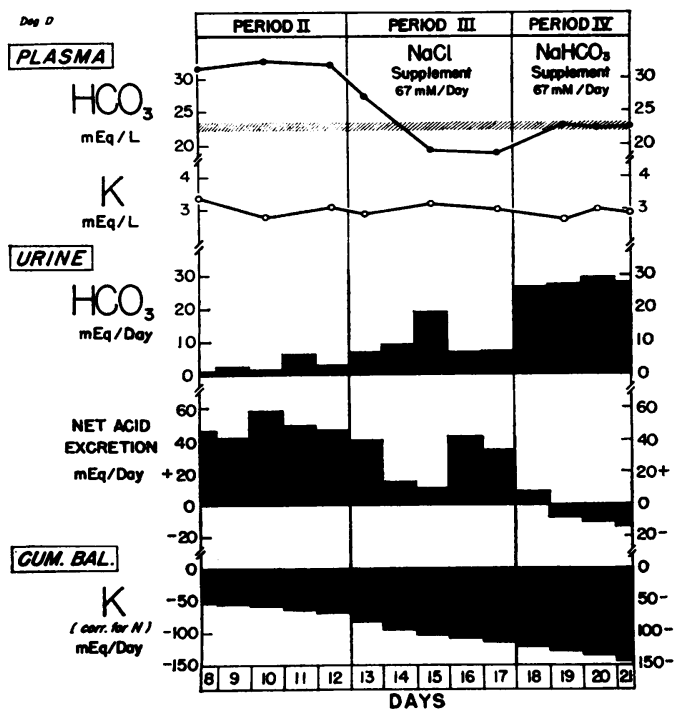

Fig. 3. EFFECT OF SODIUM BICARbonate FEEDING UPON PLASMA BICARBONATE CONCENTRATION AFTER CORRECTION of METABolic alKalosis BY SODIUM CHLORIDE (POTASSIUM-FREE DIET) (dog D).
TABLE V

Effect of sodium bicarbonate feeding upon plasma bicarbonate concentration after correction of metabolic alkalosis by sodium chloride (potassium-free diet)

\begin{tabular}{cccccc}
\hline \multicolumn{7}{c}{$\begin{array}{c}\text { Bicarb. } \\
\text { conc. } \\
\text { before } \\
\text { gastric } \\
\text { drainage }\end{array}$} & $\begin{array}{c}\text { Bicarb. } \\
\text { conc. } \\
\text { during } \\
\text { bicarb. } \\
\text { feeding }\end{array}$ & $\begin{array}{c}\Delta \text { Bi- } \\
\text { carb. } \\
\text { conc. }\end{array}$ & $\begin{array}{c}\text { Cumula- } \\
\text { tive K } \\
\text { balance }\end{array}$ & $\begin{array}{c}\text { Cumula- } \\
\text { tive Kn } \\
\text { balance }\end{array}$ \\
\hline & $m E q / L$ & $m E q / L$ & $m E q / L$ & $m E q$ & $m E q$ \\
$C-1$ & 24.1 & 24.8 & +0.7 & -136 & -132 \\
D & 22.6 & 22.8 & +0.2 & -53 & -144 \\
0 & 20.3 & 19.7 & -0.6 & -84 & -17 \\
943 & 22.7 & 24.3 & +1.6 & -74 & -217 \\
Average & 22.4 & 22.9 & +0.5 & -87 & -128 \\
\hline
\end{tabular}

bicarbonate concentration for the entire group (Protocols $\mathrm{B}$ and $\mathrm{C}$ ) fell to a level of $18.0 \mathrm{mEq}$ per $\mathrm{L}$, a decline of $13.5 \mathrm{mEq}$ per $\mathrm{L}$ from the end of Period II. This final value was $4.5 \mathrm{mEq}$ per $\mathrm{L}$ below the average control levels. In the sodium chloride animals (five studies) net acid excretion for the period fell by $102 \mathrm{mEq}$ (range, 74 to 117 $\mathrm{mEq}$ ). This decrease in renal acid excretion was reflected in a calculated loss of $15 \mathrm{mEq}$ of bicarbonate from the extracellular fluid and, by subtraction, of $87 \mathrm{mEq}$ from the intracellular fluid. A major factor in this decrement was a bicarbonate diuresis that averaged $46 \mathrm{mEq}$. The average cumulative change in the acid balance for the entire study was $+45 \mathrm{mEq}$. Dog $\mathrm{D}$, which apparently had no net loss of acid at the end of Period II, showed the same suppression of net acid excretion as did the rest of the sodium chloride group. Data obtained in one potassium chloride study showed little change in acid excretion.

2) Chloride, sodium, and potassium. As chloride was retained (Protocols $B$ and $C$ ), plasma chloride concentration rose to a final average concentration of $115 \mathrm{mEq}$ per $\mathrm{L}$, a value $6 \mathrm{mEq}$ above control. In the animals on a potassium-free diet (Protocol B) there was a further loss of potassium (corrected for $\mathrm{N}$ ) that averaged $40 \mathrm{mEq}$.

In the sodium chloride group the cumulative potassium balance (corrected for $\mathrm{N}$ ) for the entire study was $-110 \mathrm{mEq}$, cumulative sodium balance was $+108 \mathrm{mEq}$, and cumulative chloride balance was $+112 \mathrm{mEq}$. In the potassium chloride group the cumulative potassium balance (corrected for $N$ ) in the two studies was +16 and $+53 \mathrm{mEq}$. 
Period IV: sodium bicarbonate period (Figure 3 and Table $V$ )

Plasma bicarbonate concentration in the four animals rose to an average value of $23 \mathrm{mEq}$ per L. This value, although $6 \mathrm{mEq}$ per $\mathrm{L}$ above the lowest point reached during sodium chloride administration, was only $0.5 \mathrm{mEq}$ per $\mathrm{L}$ above the average control value. Frank excretion of bicarbonate occurred by the second day in each animal. The mean cumulative potassium balance at the end of the study was $-87 \mathrm{mEq}$ and corrected for nitrogen was $-128 \mathrm{mEq}$. It is of interest that in the one animal studied after alkali feeding was stopped (dog 943, which received sodium chloride throughout), plasma bicarbonate concentration promptly fell to a level well below control.

\section{Miscellaneous}

There was no significant change in the excretion of phosphate or organic acids throughout the study. Plasma creatinine concentration remained essentially unchanged. Nitrogen balance (Table I) was slightly positive in Period I but showed no striking or consistent pattern thereafter. Shifts of sodium estimated from changes in chloride space were small and variable (Table I). Body weight fell slightly during Periods I and II (cumulative average loss of $0.3 \mathrm{~kg}$ ). Stool elec- trolytes showed no significant changes. Unmeasured anion concentration rose by 4 to $5 \mathrm{mEq}$ per $\mathrm{L}$ as alkalosis developed and returned to normal during correction of the chloride deficit in Period III.

\section{Creatinine clearance studies}

Table VI presents the data on creatinine clearance during the pre- and postdrainage periods on four animals. The average rise in plasma bicarbonate concentration was $9.0 \mathrm{mEq}$ per $\mathrm{L}$. Changes in creatinine clearance were small, the values falling slightly in three instances and rising slightly in one. The mean change in clearance for the group was $-6 \%$. The possible influence of reduction in glomerular filtration rate on plasma bicarbonate concentration has been calculated on the assumption that the absolute rate of bicarbonate reabsorption would have remained constant, in a fashion similar to that observed during acute reduction of flow through the renal arteries (7). It is apparent that even in the three dogs with slight reductions in clearance, the "predicted" rise in bicarbonate falls far short of the observed rise.

\section{Discussion}

The present study demonstrates that selective depletion of hydrochloric acid leads to the development of a sustained metabolic alkalosis in ani-

TABL E VI

Creatinine clearances before and after the induction of alkalosis by gastric drainage

\begin{tabular}{|c|c|c|c|c|c|c|c|}
\hline Dog & & Control & & Alkalosis & $\begin{array}{l}\% \text { Change in } \\
\text { clearance }\end{array}$ & $\begin{array}{c}\text { "Predicted"** } \\
\text { plasma } \\
{\left[\mathrm{HCO}_{3}\right]}\end{array}$ & $\begin{array}{c}\text { Observed } \Delta \\
\text { plasma } \\
{\left[\mathrm{HCO}_{3}\right]}\end{array}$ \\
\hline & & $m l / m i n$ & & $m l / m i n$ & $\%$ & $m E q / L$ & $m E q / L$ \\
\hline M & $\begin{array}{l}67 \\
75 \\
67\end{array}$ & 70 average & $\begin{array}{l}63 \\
76 \\
86\end{array}$ & 75 average & +7 & -1.0 & +8.5 \\
\hline $\mathrm{O}$ & $\begin{array}{l}64 \\
71 \\
60\end{array}$ & 65 average & $\begin{array}{l}60 \\
52 \\
58\end{array}$ & 57 average & -12 & +2.0 & +7.5 \\
\hline $\mathrm{R}$ & $\begin{array}{l}64 \\
69 \\
70\end{array}$ & 68 average & $\begin{array}{l}66 \\
57 \\
55\end{array}$ & 59 average & -13 & +3.5 & +12.0 \\
\hline$Y$ & $\begin{array}{l}68 \\
70 \\
69\end{array}$ & 69 average & $\begin{array}{l}61 \\
68 \\
64\end{array}$ & 64 average & -7 & +1.5 & +8.5 \\
\hline
\end{tabular}

* "Predicted" $\Delta$ plasma bicarbonate is calculated on the assumption that absolute rate of bicarbonate reabsorption would remain constant despite a fall in glomerular filtration rate. 
mals maintained on a low-chloride diet. In essence, this finding indicates that chloride deficiency induced by extrarenal means leads to an elevation of the renal threshold for bicarbonate.

The events that preceded the establishment of a new steady state are, however, rather complex. During the period of gastric drainage, net acid excretion was suppressed by an amount comparable to the quantity of acid simultaneously lost from the stomach. Nevertheless, extracellular bicarbonate concentration rose by an average of $7 \mathrm{mEq}$ per L. Approximately half of this increment could be accounted for by a concomitant contraction of the extracellular fluid (assuming that the increase in alkali stores was confined solely to the extracellular space). During the drainage period there was a small loss of sodium and of potassium in the urine.

During the postdrainage period acid excretion increased above control levels by an amount that largely offset the renal retention of acid during gastric drainage. The amount of acid lost in the urine during the postdrainage phase was far more than adequate to account for the total alkali simultaneously added to the extracellular fluid.

The above description of the intermediate adjustments in acid-base balance tends to obscure some of the important over-all effects of hydrochloric acid removal that become apparent only when the new steady state is achieved. Thus, 4 to 5 days after the drainage had been discontinued, only one-fifth of the mean rise of $9 \mathrm{mEq}$ per $\mathrm{L}$ in plasma bicarbonate concentration could be accounted for by the $0.2-\mathrm{L}$ contraction of extracellular fluid; the remainder of the rise, as well as a comparable rise in tissue alkali stores, could readily be explained by the cumulative gastric and urinary acid deficit of approximately $50 \mathrm{mEq}$. This interpretation assumes, of course, that the average endogenous acid production during the entire study was not strikingly different from that of the control period, an assumption that cannot be validated with present techniques. However, the magnitude of the abrupt rise in alkali excretion during correction of the alkalosis by sodium chloride suggests that the acid balance data provide a reasonable index of alkali generation.

Regardless of the source of the alkali, the renal threshold for bicarbonate clearly must have been elevated as a consequence of chloride depletion. Since filtration rate, as estimated from exogenous creatinine clearance, was virtually unchanged from control, it can also be concluded that the rate of sodium-hydrogen exchange was markedly accelerated. As has been suggested previously (3), a deficit of reabsorbable anion, i.e., chloride, was likely responsible for the reabsorption of a larger than normal fraction of filtered sodium by exchange for hydrogen. ${ }^{3}$ The possibility cannot be dismissed, however, that the slight reduction in extracellular fluid volume (average, $0.2 \mathrm{~L}$ ) induced by chloride depletion blunted the normal postprandial rise in glomerular filtration rate and that such an alteration in renal hemodynamics impaired bicarbonate excretion. This interpretation would imply that the dog requires a postprandial elevation of glomerular filtration rate for the excretion of surplus bicarbonate, a hypothesis for which there is presently no evidence. It is also conceivable that an increase in aldosterone secretion may, in some fashion, have played a role in maintaining an elevated bicarbonate threshold.

The suppression of net acid excretion and the bicarbonate diuresis during the drainage period indicate that the rate of sodium-hydrogen exchange does not immediately rise to an extent sufficient to conserve fully the increased filtered load of bicarbonate, even when there is a concomitant deficiency of chloride. Possibly an initial period of potassium depletion is a prerequisite for full acceleration of sodium-hydrogen exchange (3). It is also possible that the sudden, marked acceleration of sodium-hydrogen exchange required for total reabsorption of the increased filtered load of bicarbonate cannot be achieved in such a short interval. The demand for rapid acceleration would be particularly great when, as in the present experiments, hydrochloric acid was removed quickly and plasma bicarbonate concentration was abruptly increased. ${ }^{4}$ There are, in fact, other circum-

\footnotetext{
3 The loss of potassium in the urine and the inability to correct the deficit of this ion while the dogs were chloride deficient can also be accounted for by a diversion of sodium to the cation exchange mechanism (3).

4 It is conceivable that if $\mathrm{HCl}$ had been removed more gradually and plasma bicarbonate concentration elevated more slowly, the loss of bicarbonate and other electrolytes would have been minimized.
} 
stances in which there is an analogous lag in the acceleration of sodium-hydrogen exchange. For example, after the administration of ammonium chloride there is a considerable initial loss of sodium and potassium, acid excretion rising markedly only after several days $(8,9)$. Similarly, in chronic hypercapnia the maximal acceleration of sodium-hydrogen exchange is delayed for at least 2 to 3 days (10).

Regardless of the complex factors considered above, it is clear that $\mathrm{HCl}$ depletion, without a concomitant removal of sodium, potassium, or water from the stomach, induces a marked metabolic alkalosis that persists for as long as the intake is kept low in chloride. These findings stand in contrast to the conventional view that some combination of factors, which include extrarenal depletion of sodium, potassium, and volume, and poor renal conservation of potassium in the face of a restricted intake, is responsible for the development of sustained alkalosis after gastric aspiration in both dog and man $(4-6,11,12)$. Whether or not man as well as dog can become alkalotic solely as the result of depletion of hydrochloric acid remains to be determined.

The data obtained during the correction phase are in accord with earlier observations that an elevated plasma bicarbonate concentration cannot be restored to normal and that potassium deficiency cannot be fully corrected without the provision of chloride $(2,3)$. Just as in previous studies, administration of sodium chloride (and a potassiumfree diet) was accompanied by a substantial reduction in net acid excretion that was more than sufficient to account for the reduction in extracellular bicarbonate stores $(2,3)$. Furthermore, when sodium bicarbonate was administered to such potassium-depleted animals, plasma bicarbonate concentration did not rise significantly above control levels; in each instance the urine became alkaline, and there was a prompt bicarbonate diuresis. The correction of alkalosis during administration of a potassium-free diet, and the failure of plasma bicarbonate concentration to rise above control values in potassium-depleted animals given sodium bicarbonate, indicate that potassium deficiency was not the critical factor in maintaining a high plasma bicarbonate concentration during the period of chloride deficiency.

\section{Summary}

Dogs were depleted of hydrochloric acid by twice daily draining their gastric contents and then promptly replacing the sodium chloride, potassium chloride, and water. Throughout the study the animals were fed a diet normal in composition except for a low-chloride content. In each instance removal of hydrochloric acid led to the development of metabolic alkalosis and a small but significant renal loss of potassium and sodium.

During the drainage period renal acid excretion was suppressed, but there was a simultaneous rise in extracellular bicarbonate concentration; approximately half of this rise could be accounted for by a contraction of the extracellular fluid. After drainage had been discontinued, renal acid excretion increased above control levels, and there was a further slight rise in plasma bicarbonate concentration. In the new steady state the total rise in bicarbonate concentration of $9 \mathrm{mEq}$ per $\mathrm{L}$ was more than adequately accounted for by the cumulative negative acid balance; the slight contraction of extracellular fluid that existed in the steady state could have explained no more than one-fifth of the increment in bicarbonate concentration.

The persistent elevation of plasma bicarbonate concentration indicates that the renal threshold for bicarbonate was significantly increased. This finding, taken together with the relatively constant creatinine clearance values, provides evidence that the absolute rate of sodium-hydrogen exchange was accelerated. These changes, as well as the acceleration of sodium-potassium exchange, can probably be attributed to a deficiency of reabsorbable anion, i.e., chloride. Administration of sodium chloride (and a potassium-free diet) quickly induced an alkali diuresis and restored normal acid-base balance.

In over-all terms the data indicate that removal of sodium, potassium, and water during gastric drainage is not a prerequisite to the development of metabolic alkalosis; to the extent that cation and water deficits develop they do so as the result of secondary renal adjustments.

\section{References}

1. Schwartz, W. B., R. M. Hays, A. Polak, and G. D. Haynie. Effects of chronic hypercapnia on electro- 
lyte and acid-base equilibrium. II. Recovery, with special reference to the influence of chloride intake. J. clin. Invest. 1961, 40, 1238.

2. Atkins, E. L., and W. B. Schwartz. Factors governing correction of the alkalosis associated with potassium deficiency; the critical role of chloride in the recovery process. J. clin. Invest. 1962, 41, 218.

3. Gulyassy, P. F., C. van Ypersele de Strihou, and W. B. Schwartz. On the mechanism of nitrateinduced alkalosis. The possible role of selective chloride depletion in acid-base regulation. $\mathrm{J}$. clin. Invest. 1962, 41, 1850.

4. Gamble, J. L., and S. G. Ross. The factors in the dehydration following pyloric obstruction. J. clin. Invest. 1924-1925, 1, 403.

5. Van Slyke, K. K., and E. I. Evans. The paradox of aciduria in the presence of alkalosis caused by hypochloremia. Ann. Surg. 1947, 126, 545.

6. Moore, F. D., E. A. Boling, H. B. Ditmore, Jr., A. Sicular, J. E. Teterick, A. E. Ellison, S. J. Hoye, and M. R. Ball. Body sodium and potassium. V. The relationship of alkalosis, potassium deficiency and surgical stress to acute hypokalemia in man. Metabolism 1955, 4, 379.
7. Thompson, D. D., and M. J. Barrett. Renal reabsorption of bicarbonate. Amer. J. Physiol. 1954, 176, 201.

8. Folling, A. On the mechanism of the ammonium chloride acidosis. Acta med. scand. 1929, 71, 221.

9. Sartorius, O. W., J. C. Roemmelt, and R. F. Pitts. The renal regulation of acid-base balance in man. IV. The nature of the renal compensations in ammonium chloride acidosis. J. clin. Invest. 1949, 28, 423.

10. Van Ypersele de Strihou, C., P. F. Gulyassy, and W. B. Schwartz. Effects of chronic hypercapnia on electrolyte and acid-base equilibrium. III. Characteristics of the adaptive and recovery process as evaluated by provision of alkali. J. clin. Invest. 1962, 41, 2246.

11. Danowski, T. S., A. C. Austin, R. C. Gow, F. M. Mateer, F. A. Weigand, J. H. Peters, and L. Greenman. Electrolyte and nitrogen balance studies in infants following cessation of vomiting. Pediatrics 1950, 5, 57.

12. Black, D. A. K., and R. P. Jepson. Electrolyte depletion in pyloric stenosis. Quart. J. Med. 1954, 23, 367. 